\title{
Tracking of secretory vesicles of PC12 cells by total internal reflection fluorescence microscopy
}

\author{
D.-M. YANG*, C.-C. HUANG†, H.-Y. LIN \\ L. -S. KAO†§, C. -W. CHI* \& C.-C. LIN \\ *Department of Medical Research and Education, Taipei Veterans General Hospital, Taipei, Taiwan, \\ China \\ $\dagger$ Institute of Biochemistry, National Yang-Ming University, Taipei, Taiwan, Republic of China \\ $\ddagger$ Department of Physics, National Taiwan University, Taipei, Taiwan, Republic of China \\ §Department of Life Science, National Yang-Ming University, Taipei, Taiwan, Republic of China \\ qDepartment of Life Sciences, Chung Shan Medical University, no. 110, Sec. 1, Chien-Kuo N. Road, \\ Taichung 402, Taiwan, Republic of China
}

Key words. Enhanced green fluorescent protein, exocytosis, fusion, membrane, Rab3, total internal reflection fluorescence microscopy, transfection, vesicle.

\section{Summary}

Total internal reflection fluorescence microscopy is used to detect cellular events near the plasma membrane. Behaviours of secretory vesicles near the cell surface of living PC1 2 cells, a neuroendocrine cell line, are studied. The secretory vesicles are labelled by over-expression of enhanced green fluorescent protein-tagged Rab3 A, one of the small G proteins involved in the fusion of secretory vesicles to plasma membrane in PC12 cells. Images acquired by a fast cooled charge-coupled device camera using conventional fluorescence microscopy and total internal reflection fluorescence microscopy are compared and analysed. Within the small evanescent range $(<200 \mathrm{~nm})$, the movements of the secretory vesicles of PC12 cells before and after stimulation by high $\mathrm{K}^{+}$are examined. The movements of one vesicle relative to another already docked on the membrane are detected. Total internal reflection fluorescence microscopy provides a novel optical method to trace and analyse the exocytotic events and vesicle specifically near a cell membrane without interference of signals from other parts of the cell.

\section{Introduction}

Amperometric and membrane capacitance measurements have been used to monitor exocytotic events during hormone and neurotransmitter release (Chow et al., 1992; Neher, 1998; Yang \& Kao, 2001). However, exocytotic events are difficult to observe directly because of the limitations of spatial and temporal resolution of conventional microscopy. Recently, total

Correspondence: Chung-Chih Lin. Tel.: 886424720022 (ext. 1804); fax: 88622823 4898; E-mail: bmlcgcsmc@hotmail.com internal reflection fluorescence microscopy (TIRFM) was used to detect fusion events near the cell surface (Neher, 1998). The advantages of TIRFM are high spatial and temporal resolution of the image, and low photo-damage to the cells (Betz\& Angleson, 1998; Neher, 1998). Based on the advanced developments of the ultrahigh numerical aperture (NA) objective lens, the current TIRFM can be used to precisely observe dynamic events occurring at the sample surface within the near-field region. The observation region is confined to a certain penetration depth which depends upon the refraction index of the sample and the incident angle of light (Axelrod, 1989).

Rab3A is member of a small G protein family expressed in neuronal and secretory cells, and is thought to be involved in regulated exocytosis. Involvement of Rab3 A protein in the fusion between secretory vesicles and plasma membrane has been proposed by Holz et al. (1994), Johannes et al. (1994) and Geppert et al. (1994). Burns et al. (1998) and Chung et al. (1999) further suggest that Rab3A is involved in the docking of vesicles with plasma membrane and recycling of secretory vesicles rather than the vesicle-plasma membrane fusion. To illustrate the key role of Rab3A, we used Rab3A fused with enhanced green fluorescent protein (EGFP), and overexpressed in PC12 cells to give bright and stable fluorescence for the direct observation of Rab3A using TIRFM. The dynamic interactions of the Rab3A-associated vesicles are then studied.

\section{Materials and methods}

Total internal reflection fluorescence microscopy

Our TIRFM, consists of an inverted epi-fluorescence microscope (IX-70; Olympus) equipped with a high numerical 
aperture lens (Apo, 60×, NA $=1.45$, Olympus). Samples are excited using the $488 \mathrm{~nm}$ line of an argon ion laser $(15 \mathrm{~mW})$ and images are acquired by a, 12-bit cooled, charge-coupled device (CCD) camera (CooISNAPf $x^{\mathrm{TM}}$ Monochrome $1300 \times$ 1030 pixels, Photometrics, Roper Scientific, Inc.) with a pixel size of $6.7 \times 6.7 \mu \mathrm{m}$. The objective is controlled by a step motor (FVX-ZM, FluoVIEW, Olympus). The image is acquired at a speed of $0.4 \mathrm{~s}$ per frame using commercial image capture software (SIMPLEPCI, Compix Inc.). To maintain good viability of the cells, the transfected cells are cultured on the cover glass, and mounted on a temperature-controlled perfusion chamber at $37^{\circ} \mathrm{C}$. The loading buffer contains $150 \mathrm{~mm} \mathrm{NaCl,} 5 \mathrm{~mm}$ $\mathrm{KCl}, 2.2 \mathrm{~mm} \mathrm{CaCl}_{2}, 1 \mathrm{~mm} \mathrm{MgCl}_{2}, 5 \mathrm{~mm}$ glucose and $10 \mathrm{~mm}$ HEPES at $\mathrm{pH} 7.4$.

\section{Cell culture}

The rat adrenal pheochromocytoma cell line, PC12, is obtained from American type culture collection (ATCC, Rockville, MD, USA). The culture condition has been described previously (Huang \& Kao, 1996). Briefly, PC12 cells are culture in Dulbecco's modified Eagle's medium containing 10\% horse serum and $5 \%$ fetal bovine serum, and incubated in an atmosphere containing $10 \% \mathrm{CO}_{2}$ at $37^{\circ} \mathrm{C}$. Cells are plated at a density of $2 \times 10^{5}$ cells per dish on poly L-lysine-coated $24 \mathrm{~mm}$ cover slips that are placed in $35 \mathrm{~mm}$ culture dishes for single-cell experiments, or $3 \times 10^{5}$ cells per well on six-well culture dishes for transfection. Each experiment is repeated at least three times with cells from different dishes and different batches.

\section{Transfection}

The day before transfection, $3 \times 10^{5}$ cells are plated into each well of six-well plates with $1 \mathrm{~mL}$ serum-containing normal growth medium without antibiotics. PC12 cells are transfected with $1 \mu \mathrm{g} \mathrm{mL}^{-1}$ DNA using $3 \mu \mathrm{g} \mathrm{mL}^{-1}$ LipofectAmine-2000 reagent as described by the manufacturer's protocol (Gibco). The cells are incubated at $37^{\circ} \mathrm{C}$ in a $\mathrm{CO}_{2}$ incubator for $6 \mathrm{~h}$ in $1 \mathrm{~mL}$ of Dulbecco's modified Eagle's medium containing 20\% horse serum and $10 \%$ fetal bovine serum. The LipofectAmine solution is then removed and replaced with fresh Dulbecco's modified Eagle's medium. The cells are incubated further for $24 \mathrm{~h}$.

\section{Stimulation set-up}

A short pulse $(0.5 \mathrm{~s})$ of high $\mathrm{K}^{+}$buffer is applied to a single cell through an ejection micropipette by a microinjection system (Picospritzer II, General Valve) with a pressure of 15 psi. The micropipette is pulled from a glass capillary by a micropipette puller (P-97, Sutter Instrument Co.). The tip of the micropipette was polished by microphorge (o.d. $2 \mu \mathrm{m}$, MF-83, Narishige). The micropipette is backfilled with $150 \mathrm{~mm} \mathrm{KCl}$ loading buffer (high $\mathrm{K}^{+}$). The ejection micropipette is positioned at a distance of $10 \mu \mathrm{m}$ from the cell.

\section{Confocal laser scanning microscopy (CLSM) and immunohistochemical staining}

The CLSM system (FluoVIEW, Olympus) was used to examine the subcellular localization of the fluorescence of overexpressed EGFP-Rab3A and immunocytochemical staining of Rab3A. Argon ion laser of wavelength of $488 \mathrm{~nm}$, and power at $5 \mathrm{~mW}$ is used as a light source to excite EGFP fluorescence. A krypton ion laser of $568 \mathrm{~nm}$ wavelength and power $5 \mathrm{~mW}$ is used to excite rhodamine fluorescence. For immunocytochemistry, EGFP-Rab3A transfected PC12 cells are fixed with paraformaldehyde, and incubated with rabbit anti-Rab3 antibody and then the rhodamine-conjugated antirabbit antibody as secondary antibody.

\section{Cytosolic calcium measurement}

PC12 cells are loaded with calcium ion sensitive dye by incubation with $5 \mu \mathrm{M}$ of the acetoxymethyl ester form of Fura-2 (Molecular Probes) for $30 \mathrm{~min}$ at $37^{\circ} \mathrm{C}$ in the loading buffer. The cover slips are washed three times with the same buffer and then examined by an inverted fluorescence microscope (IX-70, Olympus). A xenon lamp is coupled with a monochromator (Polychrome II, TILL Photonics) to provide the excitation light source. Fluorescence images of the Fura-2 excited by wavelengths of 340 and $380 \mathrm{~nm}$ are collected every $0.5 \mathrm{~s}$ by a high speed cooled CCD camera (MicroMAX: 782YHS, Princeton Instruments, Roper Scientific, Inc.). Fura-2 fluorescence ratio images acquired at an emission wavelength of $510 \mathrm{~nm}$ with alternative excitation at 340 and $380 \mathrm{~nm}$ can reveal the information of cytosolic calcium $\left(\left[\mathrm{Ca}^{2+}\right]_{\mathrm{i}}\right)$ concentration. Image acquisition and analysis are accomplished by an imaging system using a software called AXON IMAGE WORKBENCH 2.0 from Axon Instruments.

\section{Results and discussion}

We use an immunocytochemical method to identify the localization of the overexpressed EGFP-Rab3A. Figure 1(a) is a representative image of EGFP-Rab3 A transfected PC12 cells in green fluorescence. Figure $1(\mathrm{~b})$ is a representative image of rhodamine-conjugated anti-Rab3A antibody in red fluorescence, and Fig. 1(c) is the combination of Fig. 1(a), 1(b) and the DIC image. The green fluorescence of EGFP-Rab3A is colocalized with the endogenous Rab3A proteins, which is stained with an anti-Rab3A antibody. Similarly to the previous report of the localization of Rab3A in PC12 cells (Chung et al., 1999), the results of confocal images show that EGFP-Rab3A has a similar subcellular localization pattern to that of endogenous Rab3A, and most Rab3A are localized at vesicular compartments near the plasma membrane and perinuclear region.

EGFP-Rab3A over-expressed PC12 cells examined by bright field optical microscopy, epi-fluorescence microscopy and TIRFM are shown in Fig. 2. Images of the epi-fluorescence 

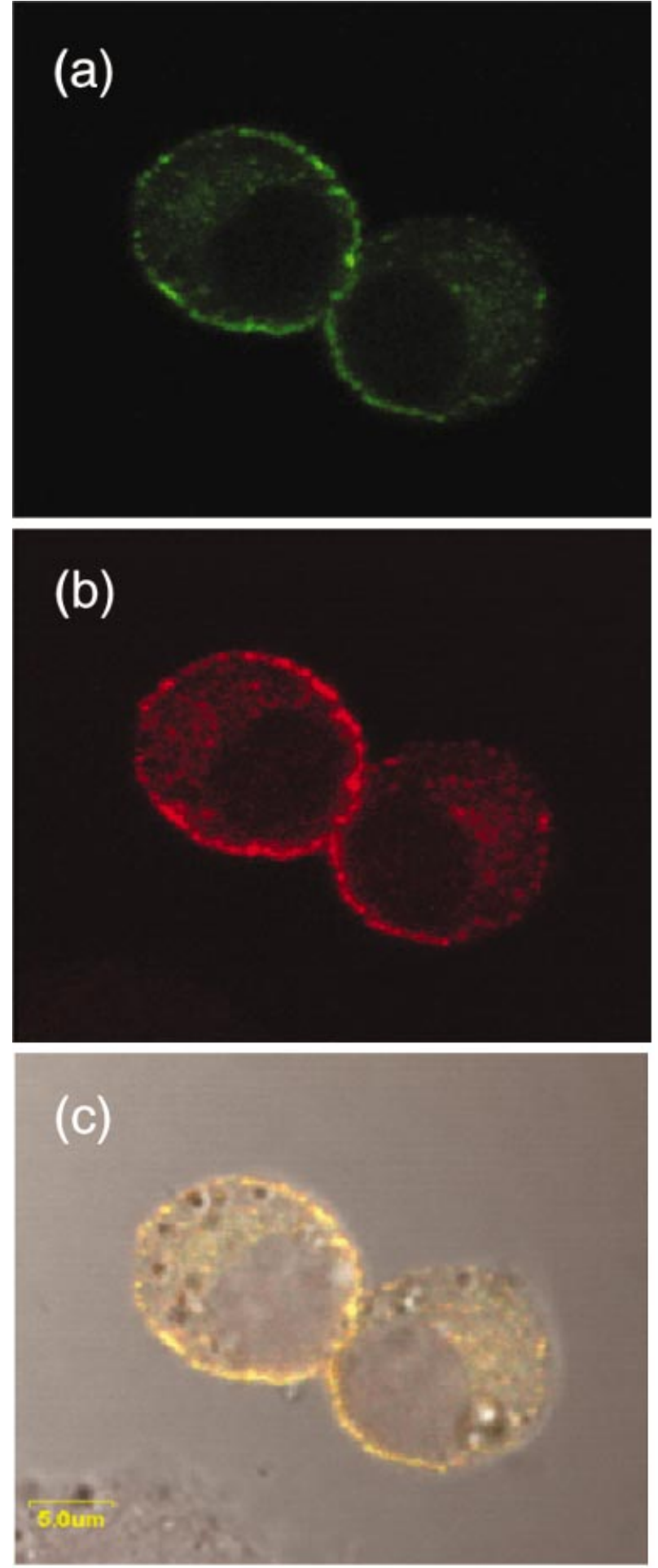

Fig. 1. Localization of Rab3A proteins expressed in PC12 cells is confirmed by the immunocytochemistry using confocal microscopy. (a) Representative image of EGFP-Rab3A transfected PC12 cells in green fluorescence. (b) Representative image of rhodamine-conjugated anti-Rab3 A antibody in red fluorescence. (c) Combination of (a), (b) and DIC image.

Fig. 2. Optical images of (a) bright field microscopy, (b) epi-fluorescence microscopy and (c) TIRFM, of the EGFP-Rab3A transfected PC12 cells.
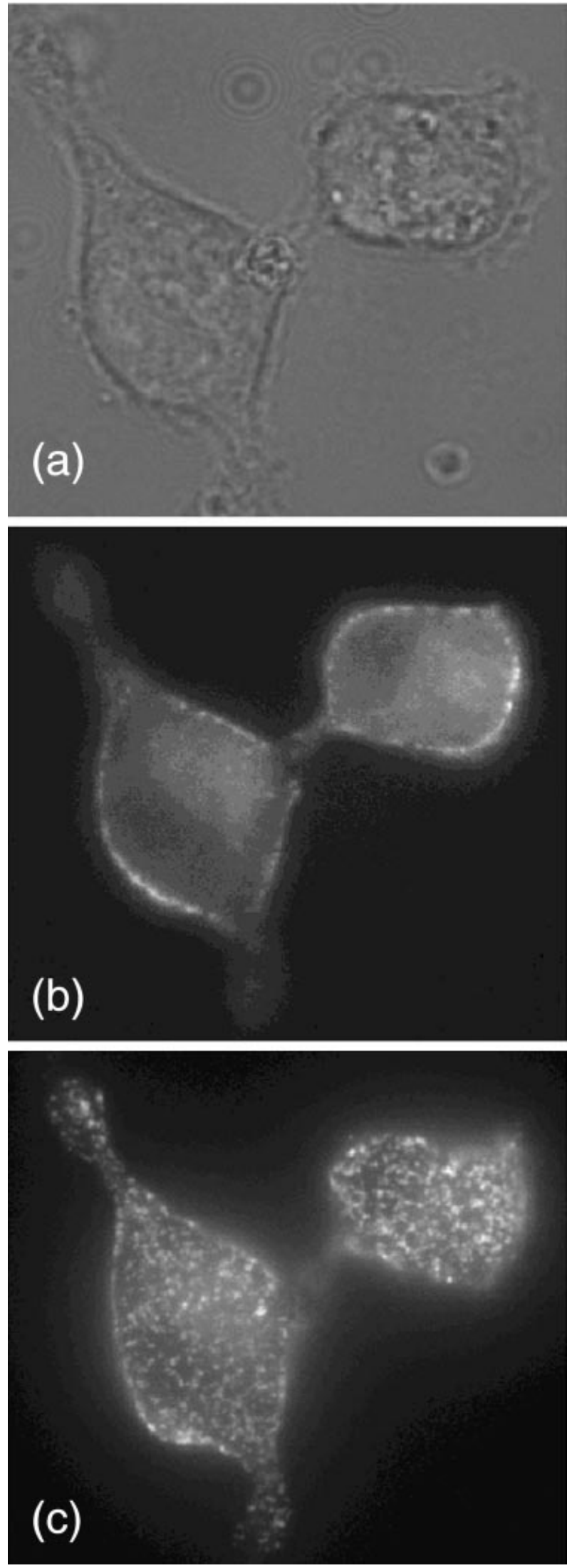


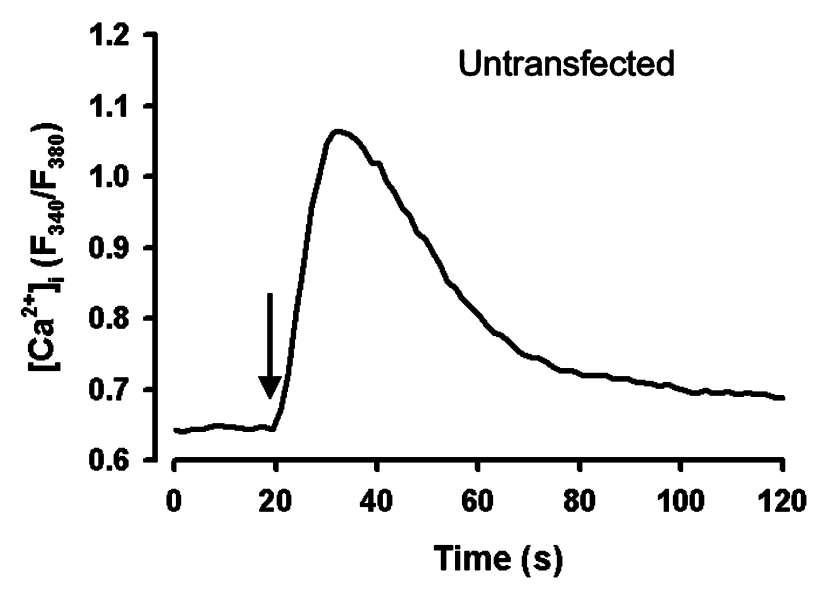

(a)

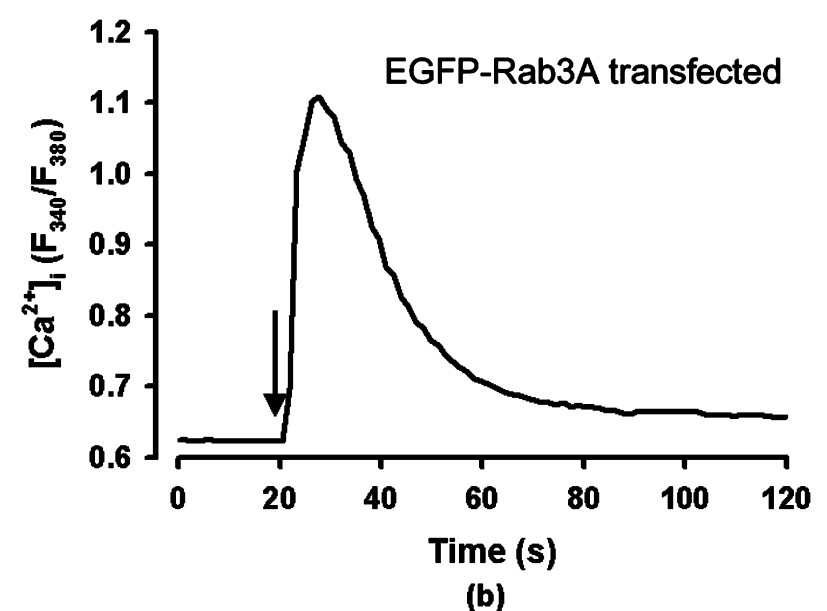

Fig. 3. Two representative cytosolic calcium data were shown. The $\left[\mathrm{Ca}^{2+}\right]_{\mathrm{i}}$ increase induced by high $\mathrm{K}^{+}$depolarization is similar between untransfected (a) and EGFP-Rab3A transfected (b) PC12 cells. Brief pulse of high $\mathrm{K}^{+}$is applied as indicated by arrows.

microscopy had the incident angle of light below the critical angle of total internal reflection, but the images of TIRFM were acquired with the incident angle larger than the critical angle of total internal reflection. Results show TIRFM can provide better resolution of the image, and the secretory vesicles labelled by EGFP-Rab3A can be discerned easily. As shown in Fig. 2(b), it is difficult for epi-fluorescence microscopy to resolve the individual vesicles that are clearly displayed in Fig. 2(c). The size of a vesicle is estimated at $220 \mathrm{~nm}$, which is in agreement with previous reports on TEM and atomic force microscopy measurements (Avery et al., 2000).

The image quality of EGFP-Rab3A acquired by TIRFM, for the time-lapse experiments is much better than that of CLSM at the same exposure time. Although the pinhole of CLSM can eliminate out-of-focus blur, it also reduces fluorescent signals, and needs longer to increase the signal-to-noise ratio. Longer exposure causes more photo-damage to fluorophores, and reduces temporal resolution.
Regulated exocytosis can be triggered by an increase in cytosolic calcium (Kao, 1988; Kao \& Cheung, 1990; Rizzuto et al., 1998; Yang \& Kao, 2001). Figure 3 displays the high $\mathrm{K}^{+}$induced cytosolic calcium elevation in cells expressing EGFPRab3A. The pattern of calcium change in these cells (Fig. 3b) was similar to that in untransfected $\mathrm{PC} 12$ cells (Fig. 3a). The increased cytosolic calcium is enough to evoke exocytosis (Yang \& Kao, 2001). The results indicate that transfection had no effect on the calcium increase in response to depolarization.

Upon stimulation by high $\mathrm{K}^{+}$, EGFP-Rab3A associated vesicles moving toward and away from the plasma membrane can be distinguished by changes in fluorescence intensity. When the vesicles move toward plasma membrane, fluorescence intensity increases because the vesicles move into the evanescent zone. Figure 4 shows an example; one vesicle moved toward the plasma membrane and appeared to fuse with a vesicle that had been docked on the membrane. With the same exposure time and experimental settings, images of EGFPRab3A can be acquired using the epi-fluorescence microscope also. However, using epi-fluorescence microscopy, it is difficult to distinguish vesicles that are near the cell surface from those that are far away from cell membrane. In addition to the study of the movement and docking of vesicles, TIRFM is also used to detect EGFP-tagged neuropeptide $Y$, a neurotransmitter in secretory vesicles. We found that fluorescence increases during docking and diffusing of vesicles, and vanishes during fusion. The secretion of neurotransmitters completes within seconds. Our system can detect fast biological events with very high temporal $(400 \mathrm{~ms})$ and spatial $(200 \mathrm{~nm})$ resolution.

\section{Conclusion}

We have successfully demonstrated the capability of resolving vesicles in living cell using TIRFM. The influence of transfection has little effect on the living cell in our experiments. The dynamics of stimulated vesicles can be clearly monitored during the exocytosis process. TIRFM provides an opportunity to trace and analyse the exocytotic events and vesicle dynamics near cell membrane without the interference of signals from out-of-focus fluorescence, and photo- and thermal damage from strong optical intensity. In future, the ability to detect the locations of Rab3A during exocytosis will help us in the further study of the role of Rab3A in regulation of exocytosis.

\section{Acknowledgements}

We thank Dr Chien-Yuan Pan for his valuable suggestions, Dr Wolfhard Almers for providing pNPY-EGFP, and Miss MingLin Hsu for her technical assistance on CLSM. The authors would like to thank Mr Shih Yuan Chiang and Dr Tashio Haneda for their technical assistance on TIRFM. This research is supported by the grants from National Science Council (NSC-89-2311-B-040-005, NSC-90-2311-B-040-006 and NSC90-2112-M-002-047), Chung Shan Medical University 


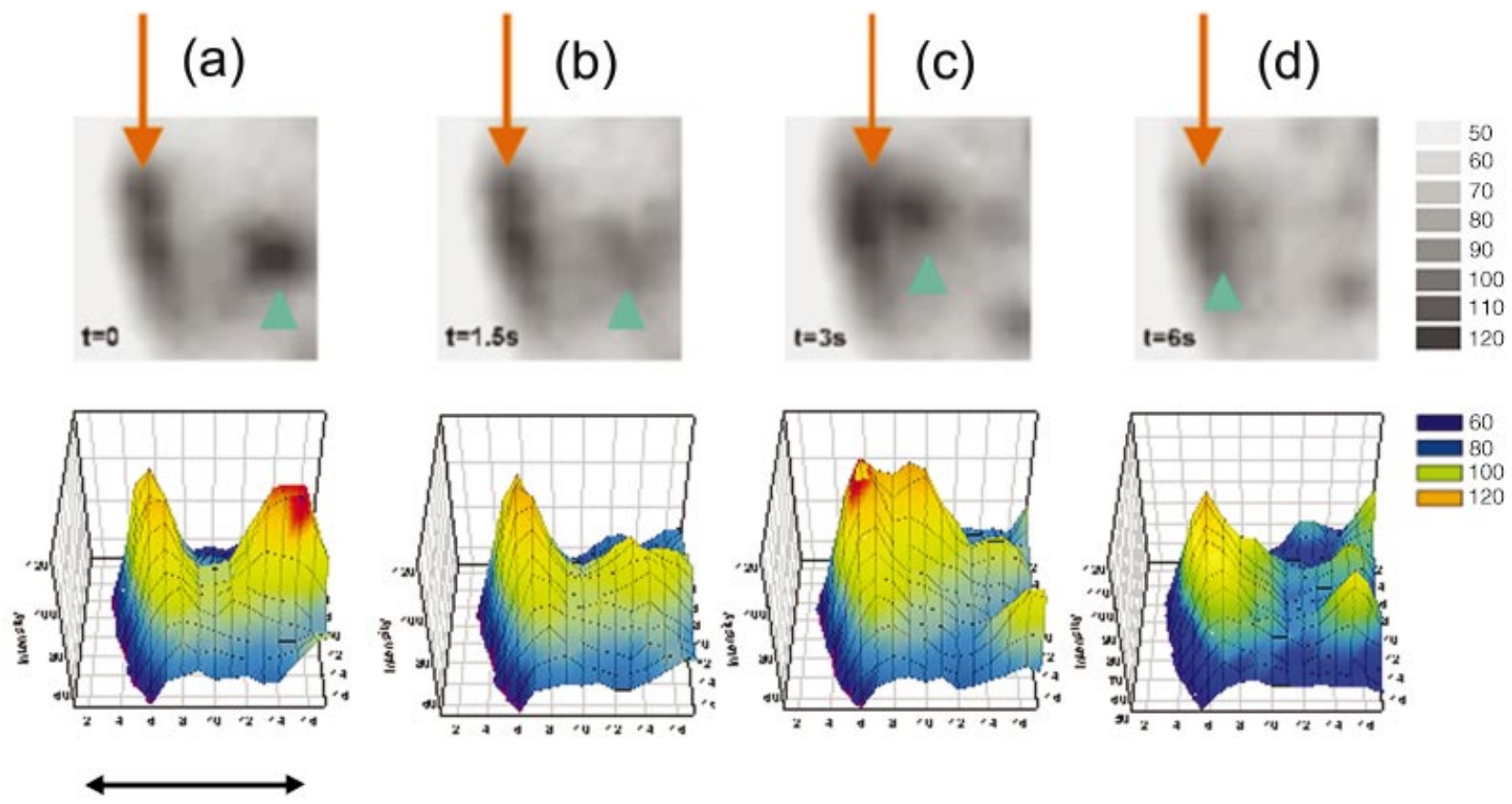

$1.23 \mu \mathrm{m}$

Fig. 4. The fusion event occurred after high $\mathrm{K}^{+}$stimulation (depolarization). EGFP-Rab3A fusion protein was over-expressed in PC12 cells. (Upper) Upon high $\mathrm{K}^{+}$stimulation, one secretory vesicle (green arrowhead in (a) $\left.t=0\right)$ was seen to first move away from the focal plane ((b) $\left.t=1.5 \mathrm{~s}\right)$, then back to the original focal plane $((\mathrm{c}), t=3 \mathrm{~s})$, and finally fused with the vesicle (red arrows) on the plasma membrane $((\mathrm{d}), t=6 \mathrm{~s})$. (Lower) $3 \mathrm{D}$ diagrams of the relative fluorescence intensity are shown.

(CSMC87-OM-B-036), Taipei Veterans General Hospital (VGH90431), and Ministry of Education (Program for Promoting Academic Excellence of Universities, Grant 89-6-FA22-2-4).

\section{References}

Avery, J., Ellis, D.J., Lang, T., et al. (2000) A cell-free system for regulated exocytosis in PC1 2 cells. J. Cell Biol. 148, 317-324.

Axelrod, D. (1989) Total internal reflection fluorescence microscopy. Methods Cell Biol. 30, 245-270.

Betz, W.J. \& Angleson, J.K. (1998) The synaptic vesicle cycle. Annu. Rev. Physiol. 60, 347-363.

Burns, M.E., Sasaki, T., Takai, Y. \& Augustine, G.J. (1998) Rabphilin-3A: a multifunctional regulator of synaptic vesicle traffic. J. Gen. Physiol. $111,243-255$.

Chow, R.H., von Rüden, L. \& Neher, E. (1992) Delay in vesicle fusion revealed by electrochemical monitoring if single secretory events in adrenal chromaffin cells. Nature, 356, 60-63.

Chung, S.H., Joberty, G., Gelino, E.A., Macara, J.G. \& Holz, R.W. (1999) Comparison of the effects on secretion in chromaffin and PC12 cells of Rab3 family members and mutants. J. Biol. Chem. 274, 18113-18120.

Geppert, M., Bolshakov, Y., Siegelbaum, S.A., Takei, K., De Camilli, P., Hammer, R.E. \& Sudhof, T.C. (1994) The role of Rab3A in neurotransmitter release. Nature, 369, 493-497.
Holz, R.W., Brondyk, W.H., Senter, R.A., Kuizon, L. \& Macara, I.G. (1994) Evidence for the involvement of Rab3A in $\mathrm{Ca}^{2+}$-dependent exocytosis from adrenal chromaffin cells. J. Biol. Chem. 269, 10229-10234.

Huang, C.-M. \& Kao, L.-S. (1996) Nerve growth factor, epidermal growth factor, and insulin differentially potentiate ATP-induced $\left[\mathrm{Ca}^{2+}\right]_{\mathrm{i}}$ rise and dopamine secretion in PC1 2 cells. J. Neurochem. 66, 124-130.

Johannes, L., Lledo, P.M., Roa, M., Vincent, J.D., Henry, J.P. \& Darchen, F. (1994) The GTPase Rab3A negative controls calcium-dependent exocytosis in neuroendocrine cells. EMBO J. 13, 2029-2037.

Kao, L.-S. (1988) Calcium homeostasis in digitonin-permeabilized chromaffin cells. J. Neurochem. 51, 221-227.

Kao, L.-S. \& Cheung, N.S. (1990) Mechanism of calcium transport across the plasma membrane of bovine chromaffin cells. J. Neurochem. 54, 1972-1979.

Neher, E. (1998) Vesicle pools and $\mathrm{Ca}^{2+}$ microdomains: new tools for understanding their roles in neurotransmitter release. Neuron, 20 , 389-399.

Rizzuto, R., Pinton, P., Carrington, W., et al. (1998) Close contacts with endoplasmic reticulum as determinant of mitochondrial $\mathrm{Ca}^{2+}$ responses. Science, 280, 1763-1766.

Yang, D.-M. \& Kao, L.-S. (2001) Relative contribution of $\mathrm{Na}^{+} / \mathrm{Ca}^{2+}$ exchanger, mitochondria and endoplasmic reticulum in the regulation of cytosolic $\mathrm{Ca}^{2+}$ and catecholamine secretion of bovine adrenal chromaffin cells. J. Neurochem. 76, 210-216. 